\title{
Clinical evaluation of a newly developed automated hemodialysis system as a clinical trial
}

Takashi Shigematsu ${ }^{1 *}$, Jun Minakuchi ${ }^{2}$, Satoshi Funakoshi ${ }^{3}$, Junichiro Hashiguchi ${ }^{4}$, Hiroshi Yoshimoto ${ }^{5}$, Kyoko $_{\text {Ito }}{ }^{6}$, Takuya Ban $^{7}$ and Tetsunosuke Kunitomo ${ }^{7}$

\begin{abstract}
Background: To cope with the increasing number of patients on long-term hemodialysis (HD), especially those with diabetic nephropathy, we designed a fully automated HD system to decrease staff workload and hence human errors related to HD treatment. In this study, we evaluated this new system as a clinical trial.

Methods: Based on a dialysis machine combined with a central dialysate delivery system (CDDS), the new system is characterized by the use of back ultrafiltrated dialysate (BUD) as a substitute fluid for priming, bonus shot and blood return, and the attachment of double endotoxin retentive filters (ETRFs).

Results: The subjects comprised 61 patients from five HD facilities enrolled in a randomized, open-labeled crossover study after giving written informed consent.

A total of $348 \mathrm{HD}$ treatments for 58 of the 61 patients were studied under a protocol designed in accordance with good clinical practice (GCP) guidelines and approved by the respective institutional review boards. No severe adverse effects were observed with either the test or control systems. The incidence of clinical events, including blood pressure decline, residual blood, and error in fluid removal, was not statistically significant in either group. Neither endotoxins nor bacteria were detected in the dialysate passing through the double filters.
\end{abstract}

Conclusions: This study confirmed the safety and effectiveness of an automated HD system based on CDDS. (This Clinical Trial No. is 21500BZZ00045000)

Keywords: Hemodialysis, Automated system, Clinical trial, Ultrafiltrated dialysate, Endotoxin retentive filter

\section{Background}

More than $95 \%$ of patients on maintenance dialysis for endstage renal disease (ESRD) in Japan undergo hemodialysis (HD). At the end of 2014, the number of such patients exceeded 320,000. The following two points are of particular importance:

1. The proportion of dialysis patients with diabetic nephropathy has been increasing steadily, reaching

\footnotetext{
* Correspondence: taki@wakayama-med.ac.jp

1 Department of Nephrology, Wakayama Medical University, Wakayama 641-8509, Japan

Full list of author information is available at the end of the article
}

nearly $45 \%$ of patients commencing dialysis and $36.6 \%$ of existing patients.

2. The population of dialysis patients is aging rapidly. For both men and women, many patients commencing dialysis are between 80 and 84 years old, representing more than 15 and $17 \%$ of those commencing dialysis, respectively, with the median age at commencement of initiating dialysis now 69.1 years $[1,2]$.

Although those trends seen in Japan are also observed worldwide, the following problems such as an increase in the number of patients requiring various 
medical cares including considerable extra support from the staffs have become more prominent in the current setting of maintenance HD in Japan.

Thus, in Japan, the dialysis staffs' shortages have become evident. The advantage of the central dialysate delivery system (CDDS) is its ability to deliver dialysis fluid to multiple patients at the same time, but full automation of the device has been required in order to decrease the number of operations, especially during the beginning and end of dialysis when multiple tasks converge.

Hemodialysis treatment is especially suitable for full automation because it involves multiple repetitions of similar operations. By implementing full automation with safety devices and fail-safe systems in place, we can achieve both the pursuit of a decrease in operational burden and an increase in efficiency, as well as safety from human error.

With the purification of dialysis fluid [3], fully automated devices actively utilizing back ultrafiltration dialysate (BUD) came into use [4]. Blood return to the arterial side is usually performed by reversing the rotation of the blood pump in a similar manner to automatic devices using physiological saline.

We developed a dialysis console using BUD, and special features of this device are that rotation of the blood pump is not reversed during blood return to the arterial side and the blood return speed can be controlled while blood return pressure is monitored. We evaluated the safety and effectiveness of this novel fully automatic dialysis machine in a clinical trial, and present the results here.

\section{Methods}

\section{Newly developed machine}

Figure 1 shows the flow scheme of a newly developed machine (TR-3000MA) as well as that of the conventional basic machine (TR-3000M) used as a control one in this study. To develop this new automated machine (TR3000MA) based upon the conventional machine (TR$3000 \mathrm{M})$, the following five features were additively designed: (1) automatic priming, (2) automatic blood removal, (3) fast fluid replacement, (4) automatic blood return, and (5) manual solution sending. To realize these function, it is noted to apply two major manipulations:

1. Non-reversed rotation of blood pump during blood return to the patient

2. Assurance of quality of back ultrafiltrated dialysate (BUD). Contrasting strikingly with the preceding machine [5], non-reversed rotation of blood pump was materialized by the device shown in Fig. 2, where blood return speed is controlled based upon the blood return pressure monitored. To control the quality of BUD, a double endotoxin (ET) retentive filter (ETRF) was placed immediately before the dialyzer on the dialysis unit side, and furthermore, the following were devised: (1) auto-flushing of the ETRF, (2) leakage test of ETRF, (3) countermeasure to failure of a single ETRF, and (4) monitoring of ETRF use duration.

\section{Study protocol}

Prior to the clinical trial, the study plan including the inclusion and exclusion criteria of patients as shown below

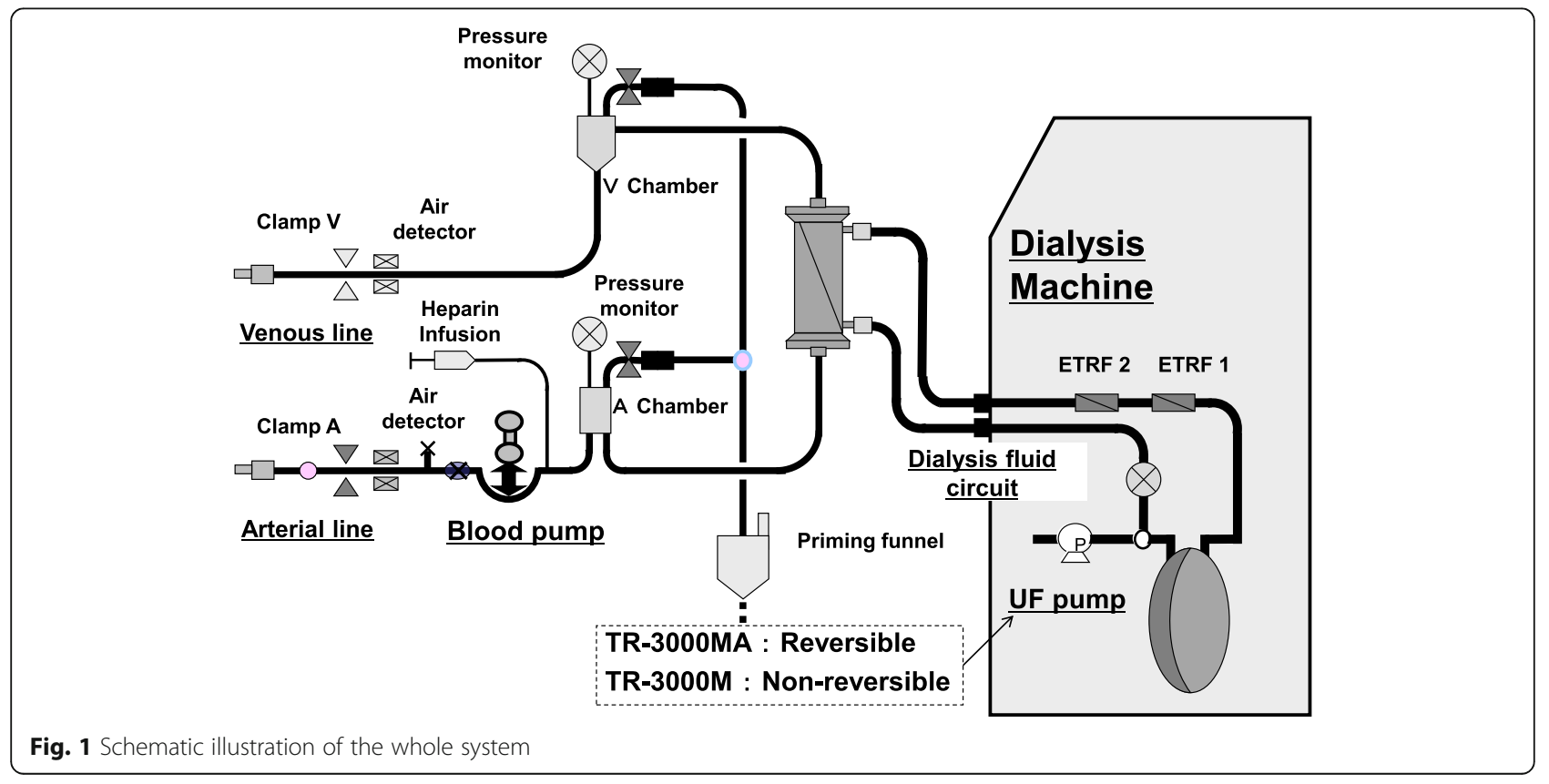




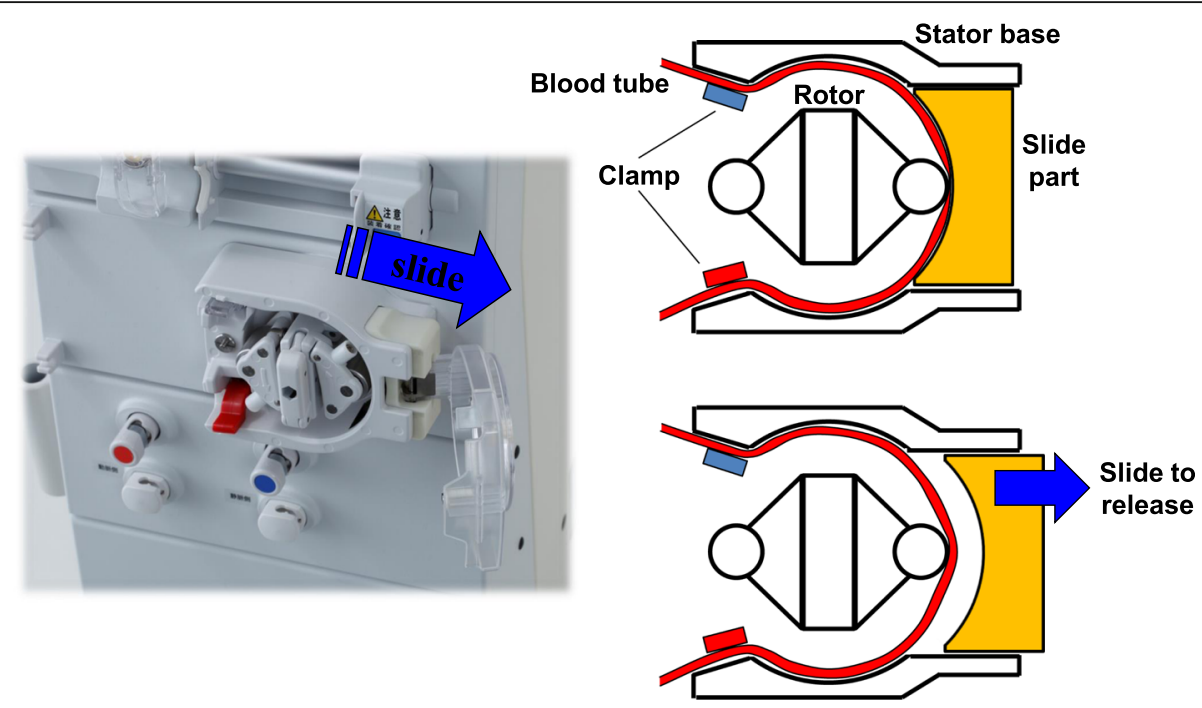

Fig. 2 A releasable blood pump. When the blood pump cover is opened, the casing slides to release the pumping unit

was reviewed by the institutional review board of each participating institution.

Inclusion criteria:

1. Duration of HD-more than 6 months

2. Age-more than 20 and less than 75

3. Treatment schedule-basically 3 to $5 \mathrm{~h}$ per treatment and three times a week

4. Physical condition-stable outpatient receiving HD as scheduled

Exclusion criteria:
1. Patients to whom kidney transplantation is planned

2. Patients to whom the shift from HD to other modalities such as HDF, CAPD, and so on is investigated

3. Patients who suffer from acute myocardial infarction, unstable angina, and cardiac insufficiency (NYHA; more than III)

4. Patients whose values of hepatic markers (AST, ALT) are higher than normal more than by two times

5. Patients who suffer from severe anemia (hematocrit; less than 20\%)

6. Patients who suffer from malignant tumors

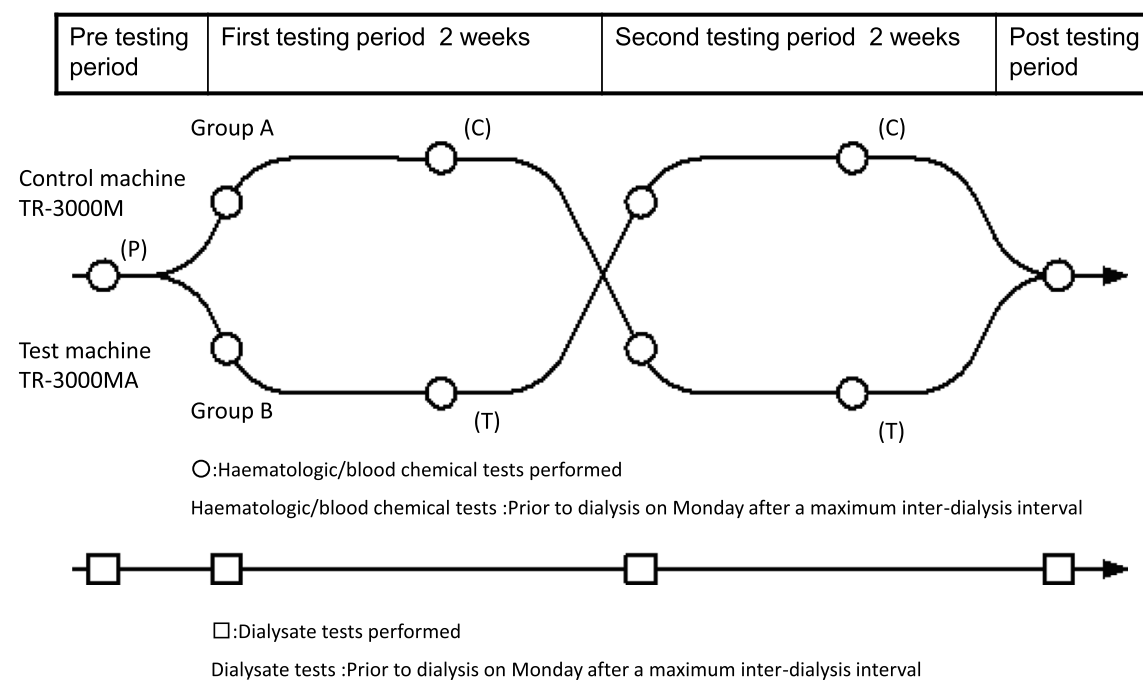

Fig. 3 Crossover diagram in this study. "T" expresses the test machine (TR-3000MA). "C" expresses the control machine (TR-3000M) 


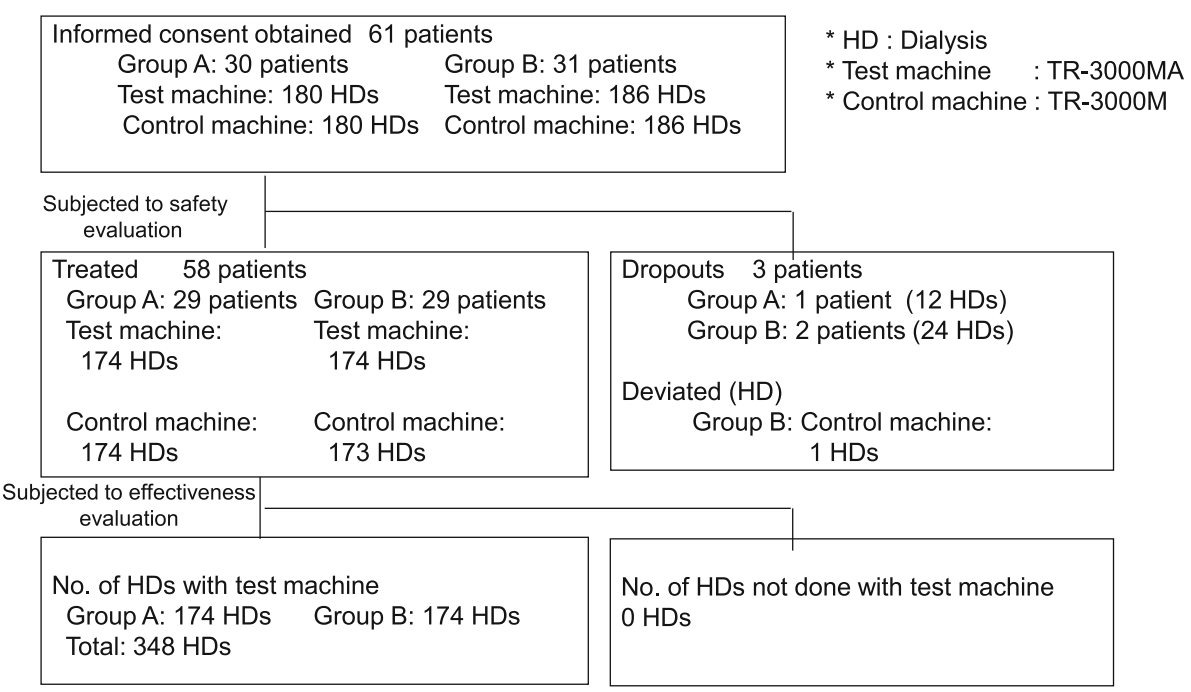

Fig. 4 Disposition of patients

7. Patients who suffer from uncontrollable hypertension or hypotension

8. Patients who are pregnant or are suspected to be pregnant

9. Patients from whom more than $150 \mathrm{~mL} / \mathrm{min}$ of extracorporeal blood volume cannot be obtained

Subjects were given an explanation of the clinical trial using the informed consent form and written information, and informed consent was given in writing by each subject. The clinical trial was conducted with due ethical considerations, such as maintaining subject confidentiality.

\section{Basic frame of protocol}

This clinical trial was an open-label, controlled crossover, non-inferiority test of TR-3000MA to the conventional machine (TR-3000M) combined CDDS. Figure 3 depicts the frame of the protocol, where long-term dialysis patients enrolled in five institutions were assigned to group A or B. Total of 60 patients were planned to be enrolled. Patients classified in group A were treated with dialysis with TR$3000 \mathrm{M}$ six times for 2 weeks and then switched to that with TR-3000MA six times for next 2 weeks, and those of group $\mathrm{B}$ were vice versa. Hematologic/blood chemical tests and biological dialysate tests were carried out at the timing shown in Fig. 3. Basically, data were compared between the phase of TR-3000MA and that of TR-3000M and analyzed mainly using the chi-square test, while patient-dependent biomarkers were analyzed using paired $t$ test.

\section{Assessment of the safety and the effectiveness}

The safety (i.e., of devices/machine and of entry of BUD into the body) and effectiveness (i.e., performance of auxiliary functions as intended) of the study test

Table 1 Background characteristics of the hemodialysis patients

\begin{tabular}{|c|c|c|c|c|}
\hline & & Group A ${ }^{* 1}$ & Group B $^{* 1}$ & Significant difference \\
\hline \multirow[t]{2}{*}{ Sex } & Male & 25 & 22 & $\mathrm{NS}^{* 2}$ \\
\hline & Female & 4 & 7 & \\
\hline \multirow[t]{2}{*}{ Age (years) } & Mean & 62.8 & 59.9 & $N S^{* 3}$ \\
\hline & SD & 8.06 & 8.99 & \\
\hline \multirow[t]{2}{*}{ Dialysis duration (years) } & Mean & 10.05 & 9.00 & $\mathrm{NS}^{* 3}$ \\
\hline & SD & 8.95 & 7.91 & \\
\hline \multirow[t]{2}{*}{ Height (cm) } & Mean & 162.21 & 161.17 & $N S^{* 3}$ \\
\hline & SD & 8.41 & 7.43 & \\
\hline \multirow[t]{2}{*}{ Dry weight (kg) } & Mean & 55.86 & 59.28 & $\mathrm{NS}^{* 3}$ \\
\hline & SD & 8.99 & 13.23 & \\
\hline
\end{tabular}

${ }^{* 1}$ Refer to Fig. 2

${ }^{*}$ Chi-square test

${ }^{*}$ Paired $t$ test 
Table 2 Hematological and laboratory results of hemodialysis patients

\begin{tabular}{|c|c|c|c|c|c|c|c|}
\hline & \multirow[t]{2}{*}{ Units } & \multirow[t]{2}{*}{ Pretesting $(P)^{* 1}$} & \multirow[t]{2}{*}{ Control machine $(C)^{* 1}$} & \multirow[t]{2}{*}{ Test machine $(T)^{* 1}$} & \multicolumn{3}{|c|}{ Significant difference (paired $t$ test) } \\
\hline & & & & & $(P)-(C)$ & $(\mathrm{C})-(\mathrm{T})$ & $(P)-(T)$ \\
\hline Leucocyte count (WBC) & $/ \mu \mathrm{L}$ & $6048.3 \pm 1950.10$ & $5974.1 \pm 1931.60$ & $6051.7 \pm 2070.96$ & NS & NS & NS \\
\hline Urea nitrogen & $\mathrm{mg} / \mathrm{dL}$ & $72.3 \pm 14.01$ & $70.7 \pm 14.50$ & $71.2 \pm 14.49$ & NS & NS & NS \\
\hline Creatinine & $\mathrm{mg} / \mathrm{dL}$ & $11.6 \pm 2.46$ & $11.5 \pm 2.43$ & $11.5 \pm 2.41$ & NS & NS & NS \\
\hline hsCRP ${ }^{* 1}$ (pre-dialysis) & $\mathrm{mg} / \mathrm{L}$ & $2114.8 \pm 3817.68$ & $2312.7 \pm 4485.56$ & $1360.1 \pm 1810.18$ & NS & NS & NS \\
\hline hsCRP ${ }^{* 1}$ (post-dialysis) & $\mathrm{mg} / \mathrm{L}$ & $2453.9 \pm 4815.46$ & $2415.4 \pm 4807.21$ & $1533.0 \pm 2115.53$ & NS & NS & NS \\
\hline
\end{tabular}

${ }^{{ }^{*} 1} h s C R P$ high-sensitivity $\mathrm{C}$-reactive protein

machine were assessed in comparison with a control one (TR-3000M) in stable-phase chronic dialysis patients for the purpose of confirming its acceptability for a plurality of operators at multiple institutions.

Safety endpoints comprised (1) occurrence of adverse events, (2) failure of the study machine, and (3) comparison of high-sensitivity C-reactive protein (CRP) values pre- and post-dialysis as a bioreactive marker to the quality of BUD infused into patients.

For adverse events for which the occurrence was foreseeable during the use of the study machine, a set of criteria was devised in advance at a clinical trial research meeting and used as the observation parameters in the clinical trial.

1) Decrease in blood pressure-fall in systolic blood pressure by $\geq 20 \%$

2) Residual blood corresponding in volume to approximately two thirds of the hollow fibers

3) Water removal error $\leq 400 \mathrm{~g}$

Failure of the study machine denoted either premature discontinuation of dialysis or completion of dialysis delayed by $1 \mathrm{~h}$ or longer related to the machine, as agreed upon at the clinical trial research meeting.

An effectiveness endpoint consisted of checking and evaluating whether the test machine had performed auxiliary functions as intended. The evaluation was made not for each patient but for each session of dialysis.

\section{Results}

The disposition of the enrolled patients and their background characteristics are shown in Fig. 4 and Table 1, respectively. There were no significant intergroup differences in respect of distribution by sex, age, dialysis duration, height, and dry weight (Table 1). As for underlying diseases, patients with chronic glomerulonephritis (33 patients, $54.1 \%$ ) tended to be more frequent and patients with diabetic nephropathy (15 patients, $24.6 \%$ ) fewer than the national mean percentages at the end of 2009 in a report of the statistical investigation.

Of the 61 patients who gave informed consent, there were 3 who withdrew consent before pre-testing, and there was a protocol deviation in one session of dialysis in group B (control machine). Consequently, 58 treated patients were subjected to the safety evaluation. For the effectiveness evaluation, 174 dialyses with the test machine in group A and 174 dialyses with the control one in group B were subjected to the assessment.

Results of the safety evaluation are summarized in Tables 2 and 3. There was no significant difference in highsensitivity CRP values because there was no significant elevation in this parameter. It was also judged that there were 57 events judged to be adversely associated with 348 dialyses using the test machine and 61 adverse events associated with 347 dialyses using the control one. There was no significant difference in the number of adverse events between the machines as a result of statistical tests of the incidence with due consideration to the number of dialyses performed. As none of the events came under the definition of failure framed in advance in association with the use of the study systems, it was judged that there was no significant difference in this respect between the test and control machines. Collectively, it was concluded that there was no difference in safety between the test and control machines.

Data especially concerning the events agreed upon at the clinical trial research meeting, among other adverse events, are presented in Table 4.

Table 3 Results of the safety evaluation (test and control machines)

\begin{tabular}{|c|c|c|c|c|c|}
\hline \multirow[t]{2}{*}{ Endpoints } & \multicolumn{2}{|c|}{ No. of adverse events } & \multicolumn{2}{|c|}{ Statistical test } & \multirow{2}{*}{$\begin{array}{l}\text { Significant } \\
\text { difference }\end{array}$} \\
\hline & Test machine & $\overline{\text { Control machine }}$ & $\overline{x^{2}}$ & $P$ value & \\
\hline Adverse event occurrence & $57^{* 1}$ & $61^{* 1}$ & 0.285 & 0.593 & NS \\
\hline Study machine failure status & $0^{* 1}$ & $0^{* 1}$ & & & NS \\
\hline Highly sensitive CRP & $0^{* 2}$ & $0^{* 2}$ & & & NS \\
\hline
\end{tabular}

${ }^{* 1}$ No. of dialyses are 348 (test machine) and 347 (control machine)

${ }^{*}$ No. of tests performed are 116 (test machine) and 114 (control machine) 
Table 4 Occurrence of adverse events (AE) agreed upon at the clinical trial research meeting and results of statistical analysis

\begin{tabular}{|c|c|c|c|c|c|c|}
\hline & Test/control machine ${ }^{* 1}$ & No. of events & Incidence (\%) & No. patients with $A E$ & Statistical test & Significant difference \\
\hline \multirow[t]{2}{*}{ Decrease in blood pressure } & Test & 24 & 6.897 & 12 & \multirow{2}{*}{$\begin{array}{l}X^{2}=0.165 \\
P=0.684\end{array}$} & \multirow[t]{2}{*}{ NS } \\
\hline & Control & 26 & 7.493 & 14 & & \\
\hline \multirow[t]{2}{*}{ Residual blood } & Test & 3 & 0.862 & 1 & \multirow{2}{*}{$\begin{array}{l}x^{2}=3.004 \\
P=0.125\end{array}$} & \multirow[t]{2}{*}{ NS } \\
\hline & Control & 0 & 0.000 & 0 & & \\
\hline \multirow[t]{2}{*}{ Water removal error } & Test & 6 & 1.724 & 6 & \multirow{2}{*}{$\begin{array}{l}X^{2}=2.295 \\
P=0.130\end{array}$} & \multirow[t]{2}{*}{ NS } \\
\hline & Control & 11 & 3.170 & 7 & & \\
\hline
\end{tabular}

${ }^{* 1}$ No. of dialyses are 348 (test machine) and 347 (control machine)

\section{Decrease in blood pressure}

A fall in blood pressure occurred during 24 dialyses with the test machine in 12 patients and during 26 dialyses with the control one in 14 patients. Of these patients, eight were common to both test and control machines. As for the circumstances in which a fall in blood pressure occurred, the event was peculiar to the study subjects, and it was found that the event occurred concomitantly with changes in water removal volume and dry weight on the day, according to the case report forms and findings by the responsible investigator. Therefore, we judged that there was no causal relationship of a decrease in blood pressure with the study machine.

\section{Residual blood}

There were three events of residual blood occurring in one patient on the test machine and no event with the control one. The events were confined to this one patient and were judged to be peculiar to this subject.

\section{Water removal error}

Water removal error occurred at 6 dialyses with the test machine in six patients and at 11 dialyses with the control one in seven patients. The results showed a significantly lower incidence for the test machine. However, the measurement of the UF pump capacity (i.e., determination of water removal precision) performed at the relevant institution on the occasion the error occurred showed no error in water removal volume, and the determination of water removal precision made after retrieval of the study machines also failed to demonstrate any error in water removal volume. As a result of the deliberation with the responsible investigators based on these considerations, the errors were thought to be attributable to errors in pre-dialysis measurement of body weight and subjects' diet adjustment. The responsible investigators and the sponsor thus judged that there was no problem with the water removal precision of the study machine.

As shown in Table 5, there were a total of 16 cases of interruption of the automatic blood return function (8 cases of air abnormality, 3 of inadequate blood pump stop position, 2 of venous pressure elevation, and 3 of arterial pressure elevation [needle tip, thrombus]). The inadequate blood pump stop position was caused by automatic blood return being started when the blood pump segment had not been fully opened. In all these events, nevertheless, the machine stopped automatically as the safety monitor functioned, and the staff manually completed blood return.

Figure 5 shows the results of water quality tests of water and dialysate samples taken at the following sites: (1) outlet of reverse osmosis (RO) equipment, (2) outlet of CDDS, (3) inlet of the dialysis machine, and (4) inlet of the dialyzer. The apparatus used at the five institutions included 7 RO equipment, 7 CDDS, and 23 dialysis machines, and the data shown represent the measurements performed four times for each site. Clinical trials were conducted after the confirmation that water/dialysate met the specified water quality standards $[6,7]$.

\section{Discussion}

As noted by the Japan Society for Dialysis Therapy [1], both the number of dialysis patients $(304,592)$ and dialysis machines $(121,835)$ showed a tendency to increase as of December 31, 2014. The status quo of the number of nurses is only being maintained. The increases in both the patient population and the patients requiring nursing care are likely to be related to the progressive increase in reported medical incidents in dialysis rooms [8, 9], with 2905 events in 2009 compared with 1946 events in 2005.

In view of the above factors, dialysis operations have been partially automated in order to prevent manipulative errors related to the increased burden on nurses, as well as to realize the practicability of concurrent care of several patients. With an automated machine, the nurse can concentrate on patient care because the dialysis

Table 5 Results of the effectiveness evaluation

\begin{tabular}{lll}
\hline Auxiliary function & $\begin{array}{l}\text { No. of completed operations/ } \\
\text { no. of operations }\end{array}$ & $\begin{array}{l}\text { Operation completion } \\
\text { rate }(\%)\end{array}$ \\
\hline Priming & $348 / 348$ & 100.0 \\
Bonus shot & $25 / 25$ & 100.0 \\
Blood return & $329 / 345$ & 95.4 \\
\hline
\end{tabular}




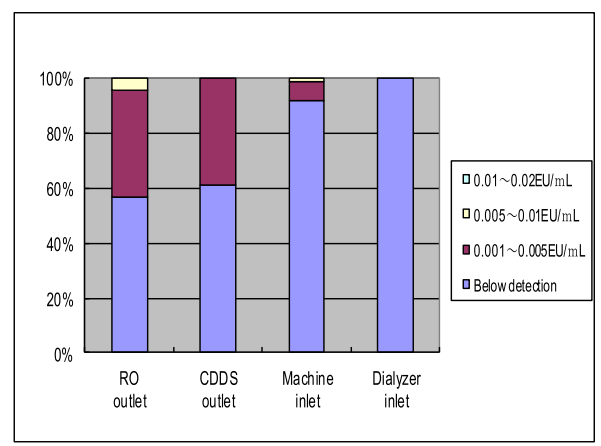

endotoxin

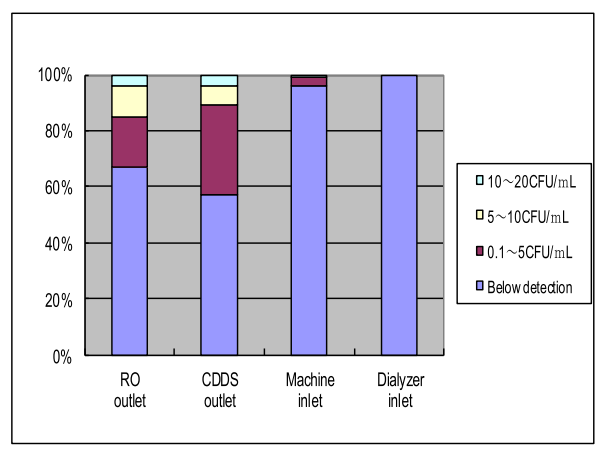

bacterial count

Fig. 5 Water quality of product water and dialysate

machine should perform safety monitoring of the intracircuit pressure, air bubbling, etc. after the nurse has programmed the machine.

The use of BUD instead of saline leads to cost reductions, as does automation of those operations that place an increased burden on staff-starting dialysis, when blood pressure falls in a patient and completion of dialysis. The lessening of workload is particularly conspicuous when CDDS is used because one staff member can prepare and initiate dialysis to several patients simultaneously. We also confirmed that the system readily fulfills the water quality control standards required for the use of BUD, and the CDDS has been judged a suitable system for producing sufficiently purified dialysate (Fig. 5).

By the way, the first clinical use of BUD was tried as "Pull and Push Therapy" by Drs. T. Shinzato and K. Maeda [10] in 1982. Although the filtration through not a dialyzer but a special filter was used, use of the filtered dialysate was approved as the substitution fluid for hemodiafiltration as Gambro's AK-100Ultra machine in 1998 [4]. As mentioned above, TR-3000MA machine was the first automated machine without the reversed rotation of blood pump during blood return, which is noted to decrease the operation risk as compared to the reversing rotation system of blood pump.

Through this clinical trial, the safety of this system was proved and later was approved by the Japanese Government. Since then, the quantitative effectiveness of this system including the labor-saving effect is being confirmed.

\section{Conclusions}

We confirmed the availability of fully automated dialysis machine by the clinical trial study.

\section{Acknowledgements}

Transparency declarations: none to declare.

I (TS) thank Ms. Yoko Doi and Midori Matsudaira for giving fine secretarial works.

\section{Funding}

This study was performed as the phase III clinical trial funded by Toray Medical Co. Ltd. Tokyo, Japan. The role of the funding body was a reward to the data collection of investigators in each chronic hemodialysis institution.

Availability of data and materials

Data are available on request to the authors.

\section{Authors' contributions}

The corresponding author (TS) planned the study, searched the literature, assessed the studies, extracted the data, analyzed the data, and prepared the article. JM, SF, JH, HY, and Kl assisted in the data collection and article preparation. TB and TK gave the detail of the study protocol from phase III clinical trial detail. All authors read and approved the final manuscript.

\section{Ethics approval and consent to participate}

The study protocol was approved by the ethical committee of the Wakayama Medical University, Wakayama, Japan. In addition, the clinical trial study protocol was also checked and approved by the institutional review board (IRB) of the Wakayama Medical University, Wakayama, Japan. Written informed consent was obtained from all subjects.

\section{Consent for publication}

All participant gave us to accept study protocol with informed consents.

\section{Competing interests}

The corresponding author (TS) has received an honorarium from Toray Medical Co. Ltd., Tokyo, Japan. TB is an employee of Toray Medical Co., Ltd., Japan. TK is a scientific advisory board in Toray Medical Co., Ltd., Japan. However, this involvement does not raise the question of bias in the work reported, or in the conclusions, implications, or opinions stated.

\section{Publisher's Note}

Springer Nature remains neutral with regard to jurisdictional claims in published maps and institutional affiliations.

\section{Author details}

${ }^{1}$ Department of Nephrology, Wakayama Medical University, Wakayama 641-8509, Japan. ${ }^{2}$ Department of Nephrology and Blood Purification, Kawashima Hospital, Tokushima, Japan. ${ }^{3}$ Department of Nephrology and Blood Purification, Nagasaki Jin Hospital, Nagasaki, Japan. ${ }^{4}$ Department of Blood Purification, Nagasaki Jin Clinic, Nagasaki, Japan. ${ }^{5}$ Division of Nephrology and Dialysis, Department of Medicine, Social Insurance Chuo General Hospital, Tokyo, Japan. ${ }^{6}$ Department of Blood Purification, Heisei Hidaka Clinic, Takasaki, Japan. ${ }^{7}$ Toray Medical Co., Ltd., Tokyo, Japan. 
Received: 5 January 2017 Accepted: 25 January 2018

\section{Published online: 09 February 2018}

\section{References}

1. Masakane I, Nakai S, Ogata S, Kimata N, Hanafusa N, Hamano T, Wakai K, Wada A, Nitta K. Annual Dialysis Data Report 2014, JSDT Renal Data Registry (JRDR). Renal Replacement Ther. 2017;3:18. https://doi.org/10.1186/s41100017-0097-8.

2. Hanafusa N, Nakai S, Iseki K, Tsubakihara Y. Japanese society for dialysis therapy renal data registry - a window through which we can view the details of Japanese dialysis population. Kidney Int Suppl (2011). 2015;5(1):15-22.

3. Koda Y, Mineshima M. Advances and advantages in recent central dialysis fluid delivery system. Blood Purif. 2009;27(Suppl 1):23-7.

4. Kawanishi H, Akiba T, Masakane I, Tomo T, Mineshima M, Kawasaki T, Hirakata H, Akizawa T. Standard on microbiological management of fluids for hemodialysis and related therapies by the Japanese Society for Dialysis Therapy. Ther Aphresis Dial. 2008;13:161-6.

5. Tsuchiya S, Moriishi M, Takahashi N, Watanabe H, Kawanishi H, KimS-T MK. Experience with the JMS fully automated dialysis machine. ASAIO J. 2003:49:547-53.

6. Kawanishi H, Moriishi M, Takahashi N, Tsuchiya S. The central dialysis fluid delivery system (CDDS): is it specialty in Japan? Renal Replace Therap. 2016;2:1. https://doi.org/10.1186/s41100-016-0016-4.

7. Tomo T, Shinoda T. Standardization of water purification in the central dialysis fluid delivery system: validation and parametric method. Blood Purif. 2009;27(Suppl 1):36-40.

8. Japanese Council for Quality Health Care. Annual Report on Medical Malpractice/Errors Information Gathering Project 2005: JCQHC. https://jcqhc.or.jp/en/.

9. Japanese Council for Quality Health Care. Annual Report on Medical Malpractice/Errors Information Gathering Project 2009 JCQHC. https://jcqhc.or.jp/en/.

10. Usuda M, Shinzato T, Sezaki R, Kawanishi A, Maeda K, Kawaguchi S, Shibata M, Toyoda T, Asakura Y, Ohbayashi S. New simultaneous HF and HD with no infuse fluid. Trans Am Soc Artif Organs. 1982;28:24-7.

\section{Submit your next manuscript to BioMed Central and we will help you at every step:}

- We accept pre-submission inquiries

- Our selector tool helps you to find the most relevant journal

- We provide round the clock customer support

- Convenient online submission

- Thorough peer review

- Inclusion in PubMed and all major indexing services

- Maximum visibility for your research

Submit your manuscript at www.biomedcentral.com/submit 